\section{PMO-128 EFFECTS OF ORAL NANOPOROUS CARBON THERAPY IN LEPTIN NULL MICE AS A MODEL OF NON-ALCOHOLIC STEATOHEPATITIS}

doi:10.1136/gutjnl-2012-302514b.128

\begin{abstract}
IJ Macnaughtan,* 'J Soeda, 'A Mouralidarane, ${ }^{2} \mathrm{~S}$ Sandeman, ${ }^{2} \mathrm{C}$ Howell, ${ }^{2} \mathrm{~S}$ Milkhalovsky, ${ }^{3} \mathrm{O}$ Kozynchenko, ${ }^{3} \mathrm{~S}$ Tennison, ${ }^{1} \mathrm{~N}$ Davies, ${ }^{1} \mathrm{R}$ Mookerjee, ${ }^{1} \mathrm{~J}$ Oben, ${ }^{1} \mathrm{R}$ Jalan. ${ }^{1}$ Hepatology, UCL, London, UK; ${ }^{2}$ Centre for Biomedical and Health Science, University of Brighton, Brighton, UK: ${ }^{3}$ MastCarbon, Guildford, UK
\end{abstract}

Introduction Endotoxaemia is implicated in the pathogenesis of nonalcoholic fatty liver disease. Modulation of intra-luminal factors driving bacterial translocation may have the capacity to impact on the natural history of the disease. Nanoporous carbons are nonabsorbable, synthetic materials which are safe with porosity manipulated for adsorption of middle and high molecular weight molecules and surface chemistry modified to alter adsorption capacity for biological molecules. We sought to determine their biological effects in leptin null mice, which are hyperphagic and obese with evident steatohepatitis and to ascertain whether nanoporous carbons can reverse established non-alcoholic steatohepatitis (NASH) in these animals.

Methods $10 \mathrm{lep}^{-} / \mathrm{lep}^{-}$null and 10 heterozygote male mice were randomised to receive powdered chow \pm carbon $(0.4 \mathrm{~g} / 100 \mathrm{~g}$ body weight/day) for 4 weeks. Extent of liver injury was assessed by serum levels of ALT. Additionally, non-parenchymal cells were isolated and the Kupffer cell (KC) population characterised by flow cytometry as those cells expressing F4/80, CD68 and CD11b. Reactive oxygen species (ROS) production by isolated KCs was also assayed. Hepatic TLR-4 expression as a surrogate of endotoxaemia was determined by immunohistochemistry.

Results In lep ${ }^{-} / \mathrm{lep}^{-}$mice, oral carbon treatment was associated with a significant reduction in ALT $889 \pm 280 \mathrm{IU} / \mathrm{ml}$ to $408 \pm 42 \mathrm{IU} /$ $\mathrm{ml}(\mathrm{p}<0.05)$. Total KC population was found to be increased in lep ${ }^{-} /$ lep ${ }^{-}$mice compared to heterozygote control with a significant reduction observed with carbon treatment $(\mathrm{p}<0.05)$. A significant reduction in KCs ROS production was also observed in carbon treated lep $\mathrm{p}^{-} / \mathrm{lep}^{-}$mice $(\mathrm{p}<0.05)$ compared to untreated lep ${ }^{-} / \mathrm{lep}$ controls. A significant reduction in the $\mathrm{F} 4 / 80+, \mathrm{CD}^{-} 8^{-}, \mathrm{CD} 11 \mathrm{~b}+$ cell sub-population in $\mathrm{lep}^{-} / \mathrm{lep}^{-}$in the presence of carbon treatment group was also observed $(p<0.05)$. Moreover, hepatic TLR-4 expression was reduced in carbon-treated lep ${ }^{-} / \mathrm{lep}^{-}$mice compared to non-treated controls. Finally, we observed a trend towards reduction in final body weight in carbon-treated lep ${ }^{-} / \mathrm{lep}^{-}$mice compared to untreated controls group $(p=0.095)$

Conclusion Oral nanoporous carbon through modulating endotoxaemia and KC function may be a promising therapy in NASH.

Competing interests None declared.

\section{PM0-129 RELAXIN REDUCES PORTAL HYPERTENSION THROUGH STIMULATION OF HEPATIC NITRIC OXIDE PRODUCTION}

doi:10.1136/gutjnl-2012-302514b.129

J Fallowfield, ${ }^{*}$ V Snowdon, R Aucott, T Gordon-Walker, A Pellicoro, J Iredale. MRC Centre for Inflammation Research, University of Edinburgh, Edinburgh, UK

Introduction We have previously reported that the multifunctional hormone relaxin (RLX) downregulated the activation state and contractility of hepatic myofibroblasts and reduced portal hypertension (PHT) in cirrhotic rats (Fallowfield J et al BASL 2010). RLX has been shown to induce a range of haemodynamic effects in different organs and species, largely through effects on nitric oxide (NO). In cirrhosis, there is hepatic NO deficiency and hyporesponsiveness. We postulated that the effects of RLX on PHTwere, at least in part, mediated by activation of the NO pathway.
Methods Cirrhosis and PHT was induced in age-matched male Sprague-Dawley rats by 8 weeks biweekly i.p. CCl4, before randomisation to the following groups: (1) recombinant human H2relaxin (H2-RLX) s.c. for $72 \mathrm{~h}$; (2) placebo s.c. for $72 \mathrm{~h}$; (3) H2-RLX s.c. + L-NAME p.o. for $72 \mathrm{~h}$; (4) placebo s.c. + L-NAME p.o. for $72 \mathrm{~h} ; n=5-10$ /group. NO levels in serum were determined by quantitative immunoassay for total nitrite and hepatic NO bioavailability by cGMP immunoassay. Relative levels of $\mathrm{Ser}^{473}$ phosphorylated Akt (p-Akt) and Ser ${ }^{1179}$ phosphorylated eNOS (p-eNOS) protein in whole liver extracts were quantified by Western blotting. Rho-kinase activity was assessed by phosphorylation of the endogenous Rho-kinase substrate moesin $\left(\mathrm{Thr}^{558}\right)$. Portal pressure (PP) and mean arterial pressure (MAP) were measured under general anaesthesia by direct cannulation.

Results Rats treated with CCl4 for 8 weeks developed micronodular cirrhosis, splenomegaly and PHT. There was no difference in mean serum nitrite levels between H2-RLX and placebo treated rats. However, H2-RLX increased hepatic cGMP production $(p<0.01)$ and upregulated expression of $\mathrm{p}$-Akt $(\mathrm{p}<0.05)$ and $\mathrm{p}$-eNOS $(\mathrm{p}<0.05)$ protein. In contrast, there was no difference in p-moesin levels. H2RLX treated animals had a lower mean PP than placebo controls $(11.6 \pm 0.3 \mathrm{~mm} \mathrm{Hg}$ [95\% CI 10.97 to 12.81$]$ vs $9.2 \pm 0.6 \mathrm{~mm} \mathrm{Hg}$ [7.66 to 10.7]; $p=0.008)$ and decreased spleen size $(p=0.01)$. The portal hypotensive effect of H2-RLX was abrogated by co-administration of the NOS inhibitor L-NAME $(11.42 \pm 0.35 \mathrm{~mm} \mathrm{Hg}$ [10.44 to 12.4]; $\mathrm{p}=0.004$ vs H2-RLX). MAP was comparable in RLX and placebo treated animals that also received L-NAME.

Conclusion A reduction in NO bioavailability is considered to be a major factor increasing intrahepatic vascular tone in cirrhosis. Our data indicate that H2-RLX was capable of stimulating intrinsic (but not systemic) NO generation in fibrotic liver by activating the Akt/ eNOS/cGMP pathway. Furthermore, inhibition of this axis with LNAME ablated the portal hypotensive effect of H2-RLX, suggesting that it could represent a novel liver-specific NO donor in cirrhotic PHT

Competing interests None declared.

\section{PM0-130 ALTERED ACETYL-COA METABOLISM IN HEPATIC MITOCHONDRIAL IMPAIRMENT IN IN VITRO MODELS OF HEPATIC CELLULAR STEATOSIS}

doi:10.1136/gutjnl-2012-302514b.130

${ }^{1} \mathrm{~K}$ Lockman, ${ }^{*}{ }^{2} \mathrm{~K}$ E Burgess, ${ }^{1} \mathrm{P}$ Lee, ${ }^{1} \mathrm{~A}$ Pryde, ${ }^{1} \mathrm{P} \mathrm{C}$ Hayes, ${ }^{3} \mathrm{C}$ Filippi, ${ }^{1} \mathrm{~J} \mathrm{~N}$ Plevris. ${ }^{1}$ Hepatology, University of Edinburgh, Edinburgh, UK; ${ }^{2}$ The Scottish Metabolomics Facility, University of Glasgow, Glasgow, UK; ${ }^{3}$ MRC Scottish Regenerative Medicine, University of Edinburgh, Edinburgh, UK

Introduction Increased ketogenesis, in the presence of unaltered $\beta$-oxidation, is a feature of human steatohepatitis. This is thought to be attributable to decreased acetyl-coA entry to tricarboxylic acid cycle with mitochondrial impairment. In this study, we examined the diversion of acetyl-coA towards free fatty acid (FFA) biosynthesis and mevalonate pathways (including vitamin D3, steroids hormones and bile acids) in the presence of mitochondrial dysfunction and triglyceride accumulation.

Methods Human hepatoblastoma C3A cells were treated with; oleate or various combinations of octanoate $(\mathrm{O})$, lactate $(\mathrm{L})$, pyruvate $(\mathrm{P})$ and ammonia $(\mathrm{N})$ for $72 \mathrm{~h}$. Metabolites that correspond to the intermediates of FFA biosynthesis, mevalonate pathways were measured using metabolomics study.

Results We have previously shown that LPON treatment, but not oleate, affected mitochondrial function as evidenced by decreased respiration and ROS formation with concomitant enhanced ketogenesis despite the similarities in triglyceride accumulation. Using metabolomics analysis, we identified three metabolites that correspond to FFA biosynthesis, three were bile acids and three were the 